\title{
Dependent Space and Attribute Reduction on Fuzzy Information System
}

\author{
Chang SHU, Zhi-Wen MO \\ College of Management Science, Chengdu university of technology, Chengdu, Sichuan, 610059, P. R. China \\ Collegeof Mathematics and Software Science, Sichuan Normal University, Chengdu, Sichuan, 610066,P.R.China \\ E-mail:20812229@qq.com ,E-mail: mozhiwen@263.net
}

\begin{abstract}
From equivalence relation $R_{B}^{\delta}$ on discourse domain $U$, we can derive equivalence relation $R^{\delta}$ on the attribute set $A$. From equivalence relation $R^{\delta}$ on discourse domain $A$, we can derive a congruence relation on the attribute power set $P(A)$ and establish an object dependent space. And then,we discuss the reduction method of fuzzy information system on object dependent space. At last ,the example in this paper demonstrates the feasibility and effectiveness of the reduction method based on the congruence relation $T^{\delta}$ providing an insight into the link between equivalence relation and congruence relation of dependent spaces in the rough set. In this way, the paper can provide powerful theoritical support to the combined using of reduction method, so it is of certain practical value.
\end{abstract}

\section{Model Basis}

Definition 1 Suppose that $(U, A, F)$ is a fuzzy information system, where $U$ is a limited object discourse domain, $A$ is a limited condition attribute set, $F$ is a mapping set. $f \in F, f: U \times A \rightarrow V, V \in 2^{[0,1]}, 2^{[0,1]}$ represents $[0,1]$ power set. $\widetilde{R}$ is a fuzzy equivalence relation on the discourse domain $U, \widetilde{R}: U \times U \rightarrow[0,1]$. Suppose that it has the following attributes:

$$
\begin{aligned}
& \text { 1) } \quad \widetilde{R}\left(x_{i}, x_{i}\right)=1 ; \\
& \text { 2) } \quad \widetilde{R}\left(x_{i}, x_{j}\right)=\widetilde{R}\left(x_{j}, x_{i}\right) ; \\
& \text { 3) } \quad \widetilde{R}\left(x_{i}, x_{j}\right) \geq \widetilde{R}\left(x_{i}, x_{k}\right) \wedge \widetilde{R}\left(x_{k}, x_{j}\right)
\end{aligned}
$$

Definition 2 Let $\widetilde{R}$ be a fuzzy equivalence relation on the discourse domain $U$, the cut relation of the fuzzy equivalence relation is defined as:

$$
R_{\delta}=\left\{\left(x_{i}, x_{j}\right) \widetilde{R}\left(x_{i}, x_{j}\right) \geq \delta\right\} \quad(0 \leq \delta \leq 1) .
$$

Theorem 1 Let $\widetilde{R}$ be a fuzzy equivalence relation on the discourse domain $U$ $R_{\delta}=\left\{\left(x_{i}, x_{j}\right) \widetilde{R}\left(x_{i}, x_{j}\right) \geq \delta\right\}(0 \leq \delta \leq 1)$ is an equivalence relation, and $\left(U, R_{\delta}\right)$ is a approximation space.

Proof . First, it is obvious that $R_{\delta}$ has reflexive and symmetric;

The following lines show the transitivity of $R_{\delta}$ :
If $\left(x_{i}, x_{k}\right) \in R_{\delta},\left(x_{k}, x_{j}\right) \in R_{\delta}$,

Then $\widetilde{R}\left(x_{i}, x_{j}\right) \geq \widetilde{R}\left(x_{i}, x_{k}\right) \wedge \widetilde{R}\left(x_{k}, x_{j}\right) \geq \delta$, where means $\left(x_{i}, x_{j}\right) \in R_{\delta}$,

Then $R_{\delta}$ has transitivity and $R_{\delta}$ is an equivalence relation, $\left(U, R_{\delta}\right)$ is approximation space.

Definition 3 Suppose that $\left(S,{ }^{\circ}\right)$ is a semi-lattice, $C: S \rightarrow S$ is a closure operator, if the following conditions hold for $\forall x, y \in S$ :

1) $x \leq C(x)$;

2) $x \leq y \Rightarrow C(x) \leq C(y)$;

3) $\quad C(C(x))=C(x)$.

Theorem 2 Let $R_{\delta}$ be an equivalence relation on, $P(U) / R_{\delta}$ is a division of $P(U)$ (discourse power set ). Suppose that $(U, A, F)$ is a fuzzy information system, $B$ is the attribute subset of attribute set of $A$,i.e. $B \subseteq A$, the subset of the discourse domain $U$,i.e. $X \subseteq U$, denote $C\left(R_{\delta}\right)$ on $(P(U), \cup)$ as:

$$
C\left(R_{\delta}\right)(X)=\bigcup\left\{Y \mid Y \in P(U) / R_{\delta}, X \cap Y \neq \phi\right\}
$$

then $C\left(R_{\delta}\right)$ is a closure operator.

Proof. It is obvious from the definition that 
1) $X \subseteq C\left(R_{\delta}\right)(X)$ holds;

2) Suppose $X_{1} \subseteq X_{2}$, we still need to prove that $C\left(R_{\delta}\right)\left(X_{1}\right) \subseteq C\left(R_{\delta}\right)\left(X_{2}\right)$.

It means that $\forall Y \in C\left(R_{\delta}\right)\left(X_{1}\right) \Rightarrow Y \in P(U) / R_{\delta} \Rightarrow X_{1} \cap Y \neq \phi \quad ; \quad$ because $X_{1} \subseteq X_{2}$, so

$X_{2} \cap Y \neq \phi \Rightarrow Y \in C\left(R_{\delta}\right)\left(X_{2}\right)$, from the arbitrariness of $Y$ we can derive $C\left(R_{\delta}\right)\left(X_{1}\right) \subseteq C\left(R_{\delta}\right)\left(X_{2}\right)$;

3) From the conclusions of 1) and 2) it is obvious that $C\left(R_{\delta}\right)(X) \subseteq C\left(C\left(R_{\delta}\right)\right)(X)$;

We still need to prove that $C\left(R_{\delta}\right)(X) \supseteq C\left(C\left(R_{\delta}\right)\right)(X)$.

For

$\forall Y \in C\left(C\left(R_{\delta}\right)\right)(X) \Rightarrow Y \in P(U) / R_{\delta} \Rightarrow Y \cap C\left(R_{\delta}\right)(X) \neq \phi \quad$, from the definition of operator we can derive $Y \cap X \neq \phi \Rightarrow Y \in C\left(R_{\delta}\right)(X)$, from the arbitrariness of $Y$ we can derive $C\left(R_{\delta}\right)(X)=C\left(C\left(R_{\delta}\right)\right)(X)$.

\section{Model of Depedent Space}

Theorem3 Let $(U, A, F)$ be a fuzzy information system , $B$ is the attribute subset of attribute set $A(B \subseteq A)$ and $R_{\delta}$ is a equivalence relation on $U$, then $R_{B}=\left\{(x, y) \mid X, Y \in P(U), C\left(R_{\delta}\right)(X)=C\left(R_{\delta}\right)(Y)\right\} \quad$ is $\quad$ a congruence relation on the semi-lattice $(P(U), U)$.

Proof . First, it is obvious from the definition that it is an equivalence relation.

Next, $X_{1}, X_{2}, Y_{1}, Y_{2} \in P(U),\left(X_{1}, X_{2}\right) \in R_{B},\left(Y_{1}, Y_{2}\right) \in R_{B}$,

From $C\left(R_{\delta}\right)\left(X_{1}\right)=C\left(R_{\delta}\right)\left(X_{2}\right), C\left(R_{\delta}\right)\left(Y_{1}\right)=C\left(R_{\delta}\right)\left(Y_{2}\right)$ we know, for $\forall Z \in P(U) / R_{\delta}$

$$
Z \cap\left(X_{1} \cup Y_{1}\right) \neq \phi \Leftrightarrow Z \cap\left(X_{2} \cup Y_{2}\right) \neq \phi
$$
$C\left(R_{\delta}\right)\left(X_{1} \cup Y_{1}\right)=C\left(R_{\delta}\right)\left(X_{2} \cup Y_{2}\right)$ holds,

It means that $\left(X_{1} \cup Y_{1}, X_{2} \cup Y_{2}\right) \in R_{B}$, it proves that $R_{B}$ is a congruence relation on $U$.

Theorem 4 Let $(U, A, F)$ be a fuzzy information system , $B$ is the attribute subset of attribute set $A(B \subseteq A), R_{\delta}$ is a equivalence relation on $U$ and $R_{B}=\left\{(x, y) \mid X, Y \in P(U), C\left(R_{\delta}\right)(X)=C\left(R_{\delta}\right)(Y)\right\}$, then $\left(U, R_{B}\right)$ is an object dependent space on $U$.

Proof. From theorem 3 we know that $R_{B}$ is a congruence relation on $U$, from definition 3 we can easily know that $\left(U, R_{B}\right)$ is object dependent space on $U$.
Definition.4 Let the $(U, A, F)$ be a fuzzy information system , $\widetilde{R}$ is fuzzy equivalence relation on discourse domain $U, \widetilde{R}: U \times U \rightarrow[0,1]$, define the relation:

$$
R_{B}^{\delta}=\left\{\left(x_{i}, x_{j}\right) \tilde{R}_{l}\left(x_{i}, x_{j}\right) \geq \delta, a_{l} \in B, B \subseteq A, x_{i}, x_{j} \in U\right\}, 0 \leq \delta \leq 1 .
$$

Theorem5 Let the $(U, A, F)$ be a fuzzy information system , $\widetilde{R}$ is a fuzzy equivalence relation on discourse domain $U, \widetilde{R}: U \times U \rightarrow[0,1]$,

$$
R_{B}^{\delta}=\left\{\left(x_{i}, x_{j}\right) \mid \tilde{R}_{l}\left(x_{i}, x_{j}\right) \geq \delta, a_{l} \in B, B \subseteq A, x_{i}, x_{j} \in U\right\}(0 \leq \delta \leq 1) \quad \text { is }
$$

an equivalence relation about attribute set $B$ on the discourse domain $U$.

\section{Proof .}

1) Reflexivity: From the definitions of $R_{B}^{\delta}$ and $\widetilde{R}$, it is obvious that $\left(x_{i}, x_{i}\right) \in R_{B}^{\delta}$;

2) Symmetry: $\left(x_{i}, x_{j}\right) \in R_{B}^{\delta} \Rightarrow\left(x_{j}, x_{i}\right) \in R_{B}^{\delta}$;

3)

$$
\begin{aligned}
& \text { Transitivity: }\left(x_{i}, x_{j}\right) \in R_{B}^{\delta}, \\
& \left(x_{j}, x_{k}\right) \in R_{B}^{\delta} \Rightarrow \widetilde{R}_{l}\left(x_{i}, x_{j}\right) \geq \delta, \widetilde{R}_{l}\left(x_{j}, x_{k}\right) \geq \delta, \\
& \text { from the theorem.1 we know that } \\
& \widetilde{R}_{l}\left(x_{i}, x_{k}\right) \geq \widetilde{R}_{l}\left(x_{i}, x_{j}\right) \wedge \widetilde{R}_{l}\left(x_{j}, x_{k}\right) \geq \delta \Rightarrow\left(x_{i}, x_{k}\right) \in R_{B}^{\delta}
\end{aligned}
$$

Theorem 6 Let $(U, A, F)$ be a fuzzy information system, $\widetilde{R}$ is fuzzy equivalence relation on discourse domain $U, \quad \widetilde{R}: U \times U \rightarrow[0,1] \quad, \quad$ denote $R^{\delta}=\left\{(X, Y) R_{X}^{\delta}=R_{Y}^{\delta}, X, Y \subseteq A\right\}$, then $R^{\delta}$ is equivalence relation on $A$.

Proof . From the definition, it is easy to know that $R^{\delta}$ is equivalence relation on $A$.

Theorem 7 Let $(U, A, F)$ be a fuzzy information system, $\widetilde{R}$ is a fuzzy equivalence relation on discourse domain

$U \widetilde{R}: U \times U \rightarrow[0,1]$
$\left.C\left(R^{\delta}\right)(X)=U\left\{Y \mid Y \in A / R^{\delta}, X \cap Y \neq \phi\right\}\right)$, then $C\left(R^{\delta}\right)$ is a closure operator on $(P(A), \cup)$.

Proof.1) Law of increasing: for $X \in P(A), x \in X$,

then

$[x]_{R^{\delta}} \bigcap X \neq \phi \Rightarrow x \in[x]_{R^{\delta}} \subseteq C\left(R^{\delta}\right)(X) \Rightarrow X \subseteq C\left(R^{\delta}\right)(X)$;

2) Monotonicity : Let $X, Y \in P(A), X \subseteq Y$, if $x \in C\left(R^{\delta}\right)(X) \Rightarrow[x]_{R^{\delta}} \cap X \neq \phi$, then

$[x]_{R^{\delta}} \cap Y \neq \phi \Rightarrow x \in[x]_{R^{\delta}} \subseteq C\left(R^{\delta}\right)(Y)$

3) From 1) and 2) we know, for $\forall X \subseteq A, C\left(R^{\delta}\right)(X) \subseteq C\left(R^{\delta}\right)\left(C\left(R^{\delta}\right)\right)(X) ;$ On the other hand: 


$$
x \in C\left(R^{\delta}\right)\left(C\left(R^{\delta}\right)\right)(X) \Rightarrow[x]_{R^{\delta}} \cap C\left(R^{\delta}\right)(X) \neq \phi, \text { from }
$$

the definition we know $[x]_{R^{\delta}} \subseteq C\left(R^{\delta}\right)(X)$,

$$
\begin{array}{ccr}
\text { It } \quad \text { mea } & x \in C\left(R^{\delta}\right)(X) & \text {,thus } \\
C\left(R^{\delta}\right)\left(C\left(R^{\delta}\right)(X) \subseteq C\left(R^{\delta}\right)(X)\right. & \text {,which } \quad \text { means } \\
C\left(R^{\delta}\right)\left(C\left(R^{\delta}\right)\right)(X) \subseteq C\left(R^{\delta}\right)(X) \text {. Thus this law is proved. }
\end{array}
$$

Theorem 8 Let $(U, A, F)$ be a fuzzy information system, $\widetilde{R}$ is fuzzy equivalence relation on discourse domain $U \quad(\quad \widetilde{R}: U \times U \rightarrow[0,1] \quad)$ then $T^{\delta}=\left\{(X, Y) \mid C\left(R^{\delta}\right)(X)=C\left(R^{\delta}\right)(Y), X, Y \in P(A)\right\}(0 \leq \delta \leq 1)$ is congruence relation on semi-lattice $(P(A), \bigcup)$.

Proof . First, from the definition it is easy to know that $T^{\delta}$ is equivalence relation.

Next,let $X_{1}, X_{2}, Y_{1}, Y_{2} \in P(A),\left(X_{1}, X_{2}\right) \in T^{\delta},\left(Y_{1}, Y_{2}\right) \in T^{\delta}$, then $C\left(R^{\delta}\right)\left(X_{1}\right)=C\left(R^{\delta}\right)\left(X_{2}\right), C\left(R^{\delta}\right)\left(Y_{1}\right)=C\left(R^{\delta}\right)\left(Y_{2}\right)$, therefore $\forall Z \in P(A) / R^{\delta}, Z \cap\left(X_{1} \cup Y_{1}\right) \neq \phi \Leftrightarrow Z \cap$ $\left(X_{2} \cup Y_{2}\right) \neq \phi$, so $C\left(R^{\delta}\right)\left(X_{1} \cup Y_{1}\right)=C\left(R^{\delta}\right)\left(X_{2} \cup Y_{2}\right)$, it means $\left(X_{1} \cup Y_{1}, X_{2} \cup Y_{2}\right) \in T^{\delta}$, thus it is proved that $T^{\delta}$ is a congruence relation on semi-lattice $(P(A), \cup)$.

Since $T^{\delta}$ is congruence relation on semi-lattice $(P(A), \cup), \quad\left(A, T^{\delta}\right)$ is called the attribute dependent space on attribute set $A$.

Note: The above deduction demonstrates that the closure operator on limited semi-lattice and congruence relation can determine each other.

\section{Attribute Reduction Based On Dependent Space}

It is feasible to discuss the issue of reducing attribute of fuzzy information system based on the attribute dependent space $\left(A, T^{\delta}\right)$ built on attribute $\operatorname{set} A$. The following is some basic knowledge of reduction theory.

$\left(A, T^{\delta}\right)$ is an attribute dependent space on the attribute set $A$ and $T^{\delta}$ is congruence relation on semilattice $(P(A), \cup)$, we denote a division of attribute power set $P(A)$ formed by congruence relation $T^{\delta}$ as $P(A) / T^{\delta}=\left\{[x]_{T^{\delta}} \mid x \in P(A)\right\}$, where $[x]_{T^{\delta}}=\left\{y \mid y \in P(A),(x, y) \in T^{\delta}\right\}$,

Then $[x]_{T^{\delta}}$ is called $\mathrm{x}$-contained congruence class of congruence relation $T^{\delta}$.

Theorem 9 We define a binary operator $\vee$ on the non-empty set $P(A) / T^{\delta}=\left\{[x]_{T^{s}} \mid x \in P(A)\right\}, \quad$ as:
$[x]_{T^{\delta}} \vee[y]_{T^{\delta}}=[x \bigcup y]_{T^{\delta}}$, then $\left(P(A) / T^{\delta}, \vee\right) \quad$ is a semi-lattice.

Proof . For $\forall[x]_{T^{\delta}},[y]_{T^{\delta}}[z]_{T^{\delta}} \in P(A) / T^{\delta}$

1) Idempotent law: $[x]_{T^{\delta}} \vee[x]_{T^{\delta}}=[x]_{T^{\delta}}$;

2) Law of commutation: $[x]_{T^{\delta}} \vee[y]_{T^{\delta}}=[x \bigcup y]_{T^{\delta}}=[y \bigcup x]_{T^{\delta}}=[y]_{T^{\delta}} \vee[x]_{T^{\delta}}$;

3) $\left.[x]_{T^{\circ}} \vee(y]_{\Gamma^{\circ}} \vee[z]_{T^{\circ}}\right)=[x]_{T^{\circ}} \vee\left([y \cup z]_{T^{\circ}}\right)=[x \cup y \cup z]_{T^{\circ}}=\left([x]_{\Gamma^{\circ}} \vee[y]_{\Gamma^{\circ}}\right) \vee[z]_{T^{\circ}}$

Definition 5 We define a binary relation $\leq$ on the non-empty set $\quad P(A) / T^{\delta}=\left\{[x]_{T^{\delta}} \mid x \in P(A)\right\}, \quad$ as: $[X]_{T^{\delta}} \vee[Y]_{T^{s}}=[Y]_{T^{s}}$, then denote it as $[X]_{T^{\delta}} \leq[Y]_{T^{\delta}}$,

Theorem $10\left(P(A) / T^{\delta}, \leq\right)$ is a join semi-lattice.

Proof . First, the binary relation $\leq$ satisfies reflexivity, anti-symmetry and transitivity, so the binary relation $\leq$ is a partial ordering relation on $P(A) / T^{\delta}=\left\{[x]_{T^{\delta}} \mid x \in P(A)\right\}$, then $\left(P(A) / T^{\delta}, \leq\right)$ is a partial ordering set.

Second, it is obvious that $[x]_{T^{\delta}} \leq[x]_{T^{\delta}} \vee[y]_{T^{\delta}} \quad, \quad[y]_{T^{\delta}} \leq[x]_{T^{\delta}} \vee[y]_{T^{s}} \quad$, if $[x]_{T^{\delta}} \leq[z]_{T^{\delta}},[y]_{T^{\delta}} \leq[z]_{T^{\delta}} \quad, \quad$ then $[x]_{T^{\delta}} \vee\left([y]_{T^{\delta}} \vee[z]_{T^{\delta}}\right)=\left([x]_{T^{\delta}} \vee[y]_{T^{\delta}}\right) \vee[z]_{T^{\delta}}=\lfloor x\rfloor_{T^{\delta}} \vee$

$[z]_{T^{\delta}}=[z]_{T^{\delta}}$, i.e. $[x]_{T^{\delta}} \vee[y]_{T^{\delta}} \leq[z]_{T^{\delta}}$, therefore there exists a least upper bound about $\leq$ in $\left\{[x]_{T^{\delta}},[y]_{T^{\delta}}\right\}$, the theorem is further proved using arbitrariness.

Definition 6 Let $(U, A, F)$ be a fuzzy information system, $T^{\delta_{1}}$ and $T^{\delta_{2}}$ are two congruence relation on the attribute power set $P(A)$. if the resultant congruence classes have the relation $[x]_{T^{\delta_{1}}} \subseteq[x]_{T^{\delta_{2}}}(x \in P(A))$, then we say that the congruence relation $T^{\delta_{1}}$ is finer than $T^{\delta_{2}}$, denoted as $T^{\delta_{1}} \leq T^{\delta_{2}}$.

Different thresholds $\delta(0 \leq \delta \leq 1)$, a number of sets formed under the congruence relation $T^{\delta}$ constitute a hierarchical congruence structure of the attribute power set $P(A)$, which is $\left\{[x]_{T^{\delta_{i}}} \mid 0 \leq \delta_{i} \leq 1\right\}$.

To better prepare the attribute reduction for information system, we need to define an order relation on the semi-lattice $(P(A), \cup)$ and create a combined semi-lattice.

Theorem 11 Let $(P(A), \mathrm{U})$ be a semi-lattice on an 
attribute power set. We define an order relation $\leq_{\vee}$ on $P(A)$ as:

$$
\begin{gathered}
\text { For } \forall x, y \in P(A), x \leq_{\vee} y \Leftrightarrow x \cup y=y, \\
\left(P(A), \leq_{\vee}\right) \text { is a join semi-lattice. }
\end{gathered}
$$

Proof. From the definition of $\leq_{\vee}$, it is easy to verify that the set satisfies reflexivity, anti-symmetry and transitivity, so $\leq_{\vee}$ is a partial order relation on $P(A)$, and $\left(P(A), \leq_{\vee}\right)$ is a partial order relation. For $\forall x, y \in P(A)$, there exists $\sup \{x, y\}$, which means $x \vee y=x \cup y$. Thus it is proved that the partial order set $\left(P(A), \leq_{\vee}\right)$ is a combined semi-lattice.

Theorem 12 Let $\left(A, T^{\delta}\right)$ be an attribute dependent space, $T^{\delta}$ is a congruence relation on the semi-lattice $(P(A), \cup)$, then for

$$
\begin{gathered}
\forall X \in P(A) / T^{\delta}, \text { let } X=\left\{x_{1}, x_{2}, \cdots x_{n}\right\} \text {, then } \\
x_{1} \cup x_{2} \cup \cdots \cup x_{n} \in X .
\end{gathered}
$$

Proof. Since $X=\left\{x_{1}, x_{2}, \cdots x_{n}\right\}$, so for $\forall i, j \in 1,2, \cdots n ., \quad\left(x_{i}, x_{j}\right) \in T^{\delta}$ holds. From the definition of congruence relation and idempotent law, $\left(x_{i}, x_{1} \cup x_{2} \cup \cdots \cup x_{n}\right) \in T^{\delta}$ holds, so it is proved that $x_{1} \cup x_{2} \cup \cdots \cup x_{n} \in X$.

Corollary1 Supposes that $\left(A, T^{\delta}\right)$ is a dependent space, $T^{\delta}$ is a congruence relation on the semi-lattice $(P(A), \cup)$, then there is a biggest element in each congruence class (equivalence class) $[x]_{T^{\delta}}$ of the congruence relation $T^{\delta}$ related to $P(A), x \in P(A)$.

Proof. Suppose congruence class (equivalence class) $[x]_{T^{\delta}}$ of the congruence relation $T^{\delta}$ related to $P(A)$ is: $[x]_{T^{\delta}}=\left\{x_{1}, x_{2}, \cdots, x_{n}\right\}$, from the theorem 12 , we can derive that $x_{1} \cup x_{2} \cup \cdots \cup x_{n} \in[x]_{T^{\delta}}$. Because $\left(P(A), \leq_{\vee}\right)$ is a combined semi-lattice, therefore $x_{1} \vee x_{2} \vee \cdots \vee x_{n}=x_{1} \cup x_{2} \cup \cdots \cup x_{n} \in[x]_{T^{\delta}}$ $x_{1} \vee x_{2} \vee \cdots \vee x_{n}$ is the biggest element in $[x]_{T^{\delta}}$.

Note: The corollary1 indicates that for different congruence structures, there is always a biggest element in each congruence class. This provides a theoretical basis for finding attribute reduction method using congruence relation.

Theorem 13 Let $\left(A, T^{\delta}\right)$ be a dependent space , where $T^{\delta}$ is a congruence relation on the semi-lattice $(P(A), \bigcup)$. For $\forall x \in P(A)$, then $C\left(T^{\delta}\right)(x)=\bigcup[x]_{T^{\delta}}$ is a closure operator on $(P(A), \cup)$.

Proof. The method is similar to the proof of theorem 7.

Definition.7 Let $\left(A, T^{\delta}\right)$ be a dependent space , where $T^{\delta}$ is a congruence relation on the semi-lattice $(P(A), \cup), C\left(T^{\delta}\right)$ is a closure operator on $(P(A), \cup)$, denote the set made up of all $C\left(T^{\delta}\right)$ closure elements in $P(A)$ as $C_{T^{\delta}}$, then

$$
\forall x \in C_{T^{\delta}} \Rightarrow C\left(T^{\delta}\right)(x)=x
$$

Theorem 14 Let $\left(A, T^{\delta}\right)$ be a dependent space, where $T^{\delta}$ is a congruence relation on the semi-lattice,$(P(A), \mathrm{U})$ denote

$$
T_{\delta}^{L}=\left\{(x, y) \mid x, y \in P(A), \forall z \in C_{T^{\delta}}, x \leq_{\wedge} z \Leftrightarrow y \leq_{\wedge} z\right\} \text {, where }
$$

$T_{\delta}^{L} \quad$ is a congruence relation on semi-lattice $(P(A), \cup)$, and $T_{\delta}^{L}=T^{\delta}$.

Proof. Since $\left|C_{T^{\delta}}\right|=\left|P(A) / T^{\delta}\right|$, and $\forall z \in C_{T^{\delta}}, z$ is the biggest element in the congruence class $T^{\delta}$ related to $P(A)$ so $\forall x, y \in P(A) \Rightarrow(x, y) \in T^{\delta} \Leftrightarrow(x, y) \in T_{\delta}^{L} \Rightarrow T_{\delta}^{L}=T^{\delta}$, thus we prove that it is a congruence relation.

In the following discussion, $\sigma(D)$ represents the smallest algebraic unit created by dividing $D$.

Theorem 15 Let $(U, A, F)$ be a fuzzy information system, there is an attribute dependent space $\left(A, T^{\delta}\right), \forall x, y \in A, \forall X \subseteq A, B \subseteq U$, the following conclusions can be drawn:

1) $(x, y) \in R^{\delta} \Leftrightarrow(\{x\},\{y\}) \in T^{\delta}$;

2) $\quad X \in A / R^{\delta} \Rightarrow X \in C_{R^{\delta}}$;

3) $C_{R^{\delta}}=C_{T^{\delta}}=\sigma\left(A / R^{\delta}\right)$.

\section{Proof.}

1) Because

$$
\begin{aligned}
& (x, y) \in R^{\delta} \Leftrightarrow[x]_{R^{\delta}}=[y]_{R^{\delta}} \Leftrightarrow C\left(R^{\delta}\right)(\{x\})=C\left(R^{\delta}\right)(\{y\}) \\
& \Leftrightarrow(\{x\},\{y\}) \in T^{\delta}
\end{aligned}
$$

2) From the definition of closure operator, it is obvious that the conclusion holds;

3) Prove $C_{R^{\delta}}=C_{T^{\delta}}$ at first:

Suppose

$$
X \in C_{R^{\delta}}, \forall Z \in P(A), \quad \text { if } \quad X \subset Z \quad,
$$

$C\left(R^{\delta}\right)(X)=\bigcup\left\{Y \mid Y \in A / R^{\delta}, Y \cap X=\phi\right\}=X$ 
There exists $Y \in A / R^{\delta}$, thatmakes $Y \cap(Z-X) \neq \phi$, it leads to $C\left(R^{\delta}\right)(Z)=\bigcup\left\{Y \mid Y \in A / R^{\delta}, Y \cap(X \bigcup(Z-X))\right.$

$$
\neq \phi\}=X \bigcup\left(\bigcup\left\{Y \mid Y \in A / R_{R^{\delta}}, Y \cap(Z-X) \neq \phi\right\}\right) \neq X
$$

, so, for $\forall Y \in[X]_{T^{\delta}}, Y \subseteq X$, it leads to

$$
X=\bigcup[X]_{T^{\delta}} \in C_{T^{\delta}} \text {, thus } C_{R^{\delta}} \subseteq C_{T^{\delta}}
$$

Next we prove $C_{R^{\delta}} \supseteq C_{T^{\delta}}$.Suppose $X \in C_{T^{\delta}}$ ， because $X=\bigcup[X]_{T^{\delta}}$, so $X$ is the biggest element in the congruence class $[X]_{T^{\delta}}$, suppose there exists $Y \in A / R^{\delta}, Y \not \subset X, Y \cap X \neq \phi$, which means $Y \subseteq C\left(R^{\delta}\right)$ $(X)$.

Therefore there exists $Z \in P(A), Z \not \subset X$ that makes $C\left(R^{\delta}\right)(X)=C\left(R^{\delta}\right)(Z)$, this means $Z \in[X]_{T^{\delta}}$, this is contradictory to the statement that $X$ is the biggest.

So for

$$
\forall Y \in A / R^{\delta}, Y \cap X \neq \phi \quad C_{R^{\delta}}, \text { then } Y \subseteq X \text {, that }
$$

means $X=\bigcup\left\{Y \mid Y \in A / R^{\delta}, Y \cap X \neq \phi\right\} \in C_{R^{\delta}}$,

So $C_{R^{\delta}} \supseteq C_{T^{\delta}}$. Thus $C_{R^{\delta}}=C_{T^{\delta}}$ is proved.

Next we prove $C_{R^{\delta}}=\sigma\left(A / R^{\delta}\right)$.

From $C_{R^{\delta}}(A)=A$, it is easy to get to $A \in C_{R^{\delta}}$. Suppos $\quad X, Y \in C_{R^{\delta}} \quad$ then

$$
\begin{aligned}
& X \cup Y=\left\{\bigcup\left\{Z \mid Z \in A / R_{R^{\delta}}, Z \cap X \neq \phi\right\}\right. \\
& \cup\left(\cup\left\{Z \mid Z \in A / R^{\delta}, Z \cap Y \neq \phi\right\}\right)=\bigcup\left\{Z \mid Z \in A / R^{\delta}, Z \cap(X \cup Y) \neq \phi\right\}=C\left(R^{\delta}\right)(X \cup Y) \in C_{R^{\delta}}
\end{aligned}
$$$$
\text { , further } \forall Y \in A / R^{\delta}, Y \cap X \neq \phi \Leftrightarrow Y \subseteq X \Leftrightarrow Y \cap \sim
$$

$\mathrm{X}=\phi$, which means $Y \cap \sim \mathrm{X} \neq \phi \Leftrightarrow Y \cap X=\phi$.

Therefore

$$
C\left(R^{\delta}\right)
$$

$\mathrm{X})=$

$\cup\left\{Y \mid Y \in A / R^{\delta}, Y \cap(A-X) \neq \phi\right\}=\bigcup\left\{Y \mid Y \in A / R^{\delta}, Y \cap X=\phi\right\}=\sim$ $\mathrm{X} \in C_{R^{\delta}}$, so $C_{R^{\delta}}$ is the smallest algebraic unit generated by $A / R^{\delta}$, thus the equation is proved.

Next, we discuss dependent relation which is unique in attribute dependent space.

Definition.8 Given a fuzzy information system $(U, A, F)$, for attribute dependent space $\left(A, T^{\delta}\right)$, for $X \subseteq A$, if $C\left(T^{\delta}\right)(A) \subseteq C\left(T^{\delta}\right)(X)$, we say that the attribute set $A$ is dependent on the attribute subset $X$, denoted as $(A, X) \in d r$ (i.e. $A$ is dependent on $X$ ).

Definition 9 Let $\left(A, T^{\delta}\right)$ be an attribute dependent space, we call $X \subseteq A$ is the reduction set of $A$. If the following conditions are satisfied:

1) $(A, X) \in d r$;

2) $\forall Y \subset X,(A, Y) \notin d r$.

Theorem 16 Let $(U, A, F)$ be a fuzzy information system, for attribute dependent space $\left(A, T^{\delta}\right)$ and $X \subseteq A$, if $(A, X) \in d r$, then the smallest set $X$ (based on set inclusion relation) that satisfies $(A, X) \in d r$ is the reduction set of the attribute set $A$.

Proof - Because $\forall X \subseteq A \quad, \quad$ so $(A, X) \in d r \Leftrightarrow C\left(T^{\delta}\right)(A)=C\left(T^{\delta}\right)(X) \Leftrightarrow(A, X) \in T^{\delta}$, thus the theorem is proved.

Definition 10 Let $(U, A, F)$ be a fuzzy information system, for attribute dependent space $\left(A, T^{\delta}\right)$, if $(A, X) \in d r$, then $d d=\frac{\left|C\left(T_{B}^{\delta}\right)(A)\right|}{\left|C\left(T_{B}^{\delta}\right)(X)\right|}$ is the degree of dependence of the attribute set $A$ on attribute subset $X$, where $|\bullet|$ represents the number of set elements.

The degree of dependence describes the degree to which the attribute set $A$ depends on the attribute subset $X$. The degree of dependence has the following characteristics:

1) $0 \leq d d \leq 1$;

2) The bigger the $d d$ is, the higher the degree to which the attribute set $A$ depends on the attribute subset $X$, indicating that the attribute subset $X$ is more important. The smaller the $d d$ is, the lower the degree to which the attribute set $A$ depends on the attribute subset $X$, indicating that the attribute subset is less important.

We can see from this that the reduction of the attribute set $A$ can also be described as finding the attribute subset that makes the degree of dependence $d d \in(0,1)$ reach the maximum value.

Next, given the congruence relation in the attribute dependent space, a system of attribute congruence classification (division) as well as a theory about attribute reduction and some reduction methods will be put 
forward.

Definition 11 Let $(U, A, F)$ be a fuzzy information system, and $\left(A, T^{\delta}\right)$ an attribute dependent space, where $T^{\delta}$ is a congruence relation on the attribute power set $P(A), \quad M \in P(A)$ is the smallest element (based on set inclusion relation) in $[X]_{T^{\delta}}$, then $M$ is $T^{\delta}$ representative element in $[X]_{T^{\delta}}$, the set made up of all representative elements of congruence class as $E\left(T^{\delta}\right)$.

Lemma 1 Let $(U, A, F)$ be a fuzzy information system, attribute dependent space $\left(A, T^{\delta}\right), T^{\delta}$ is a congruence relation on the attribute power set $P(A)$, for $X, Y \in P(A) \quad, \quad$ if $\quad Y \subseteq X$, and $X \in E\left(T^{\delta}\right), \quad$ then $Y \in E\left(T^{\delta}\right)$.

Proof . If $Y \notin E\left(T^{\delta}\right)$, then $Y \neq X$, and $\exists Z \in[Y]_{T^{\delta}}, Z \in E\left(T^{\delta}\right)$, then $Z \subseteq Y, Z \neq Y$, from $(Z$, $Y) \in T^{\delta}$ then $(Z \cup(X-Y), X)=(Z \cup(X-Y), Y \bigcup(X-Y)) \in T^{\delta}$ Moreover, because $Z \cup(X-Y) \subseteq X, Z \cup(X-Y) \neq X$, is contradictory to $X \in E\left(T^{\delta}\right)$, thus the lemma is proved.

Definition 12 If $Y \subseteq A,(Y, A) \in T^{\delta}$, and $\forall y \in Y$, then $(Y-y, A) \notin T^{\delta}$. We call $Y$ as a $T^{\delta}$-reduction of attribute set $A$.

The question now is to choose an attribute reduction that satisfies the conditions from all the subsets of the attribute set $A$. We know if there are $N$ attributes in the attribute set $A$, then the number of its non-empty subsets is $2^{N}-1$, because the attribute set is a limited set, so the reduction set can be found for certain using definition 12. However, usually there are multiple reductions.

All the $T^{\delta}$-reduction sets $\left\{Y_{i}: i \leq l\right\}$ of $A$ are denoted as reduct $\left(A, T^{\delta}\right)$. If the intersection set of all $T^{\delta}$ - reduction sets of $A \quad Y=\bigcap\left\{Y_{i}\right\}$ is non-empty, we call $Y$ the core attribute in the attribute set $A$, denoted as $\operatorname{CORE}(A)$. But usually the core attribute does not constitute the reduction of attribute. All reductions contain core attribute, therefore core attribute is absolutely necessary. The attributes in $\bigcup\left\{Y_{i}\right\}-\bigcap\left\{Y_{i}\right\}$ do not appear in all reductions therefore they are relatively necessary. The attributes in $A-\bigcup\left\{Y_{i}\right\}$ do not appear in any reduction therefore are absolutely unnecessary.

\begin{tabular}{|c|c|c|c|c|}
\hline & $x_{1}$ & $x_{2}$ & $x_{3}$ & $x_{4}$ \\
\hline$a$ & 0.5 & 0.6 & 1 & 1 \\
\hline$b$ & 1 & 0.7 & 0.9 & 0.9 \\
\hline$c$ & 0.7 & 1 & 1 & 0.9 \\
\hline$d$ & 0.5 & 0.5 & 0.1 & 0.1 \\
\hline
\end{tabular}

The procedure of attribute reduction for fuzzy information system under the dependent space model is as follows:

Step 1 Work out the congruence relation $T^{\delta}$ based on the given fuzzy information system $(U, A, F)$ and choose the threshold $\delta$ (the method of choosing $\delta$ is the same as the method in example 1 where $F$ distribution is used).

Step 2 Work out the congruence classification $P(A) / T^{\delta}$ of $P(A)$ based on the chosen congruence relation.

Step 3 Find out the smallest element set $M\left(T^{\delta}\right)$ (based on smallest elements with inclusion relation) of each congruence class in all congruence classes.

Step 4 Write out the set made up of all $T^{\delta}$-reduction sets reduct $-\left(A, T^{\delta}\right)$.

Step 5 Work out the intersection set of all reduction sets. If the intersection set is not empty, it is the core attribute $\operatorname{set} \operatorname{CORE}(A)$. If the intersection set is empty, there is no core attribute.

Example 1 The table below defines a fuzzy information system

\section{Table 1 fuzzy information system}

Let $\delta_{1}=0.1, \delta_{2}=0.5, \delta_{3}=0.6, \delta_{4}=0.7, \delta_{5}=0.9, \delta_{6}=1$, we can obtain the cut relation matrix and the equivalence classes of the attribute set $A$ :

$$
\begin{aligned}
& \left(\begin{array}{llll}
1 & 1 & 1 & 1 \\
1 & 1 & 1 & 1 \\
1 & 1 & 1 & 1 \\
1 & 1 & 1 & 1
\end{array}\right) \delta_{1}=0.1, A / R^{\delta_{1}}=\{a b c d\},\left(\begin{array}{llll}
1 & 1 & 1 & 1 \\
1 & 1 & 1 & 1 \\
1 & 1 & 1 & 1 \\
1 & 1 & 0 & 0
\end{array}\right) \\
\delta_{2} & =0.5, A / R^{\delta_{2}}=\{a b c, d\}
\end{aligned}
$$

\section{Methods And Examples Of Attribute Reduction}




$$
\begin{aligned}
& \left(\begin{array}{llll}
0 & 1 & 1 & 1 \\
1 & 1 & 1 & 1 \\
1 & 1 & 1 & 1 \\
0 & 0 & 0 & 0
\end{array}\right) \delta_{3}=0.6, A / R^{\delta_{3}}=\{a, b c, d\}, \\
& \left(\begin{array}{llll}
0 & 0 & 1 & 1 \\
1 & 1 & 1 & 1 \\
1 & 1 & 1 & 1 \\
0 & 0 & 0 & 0
\end{array}\right)_{\delta_{4}}=0.7, R^{\delta_{4}}=\{a, b c, d\} \\
& \left(\begin{array}{llll}
0 & 0 & 1 & 1 \\
1 & 0 & 1 & 1 \\
0 & 1 & 1 & 1 \\
0 & 0 & 0 & 0
\end{array}\right) \delta_{5}=0.9, R^{\delta_{5}}=\{a, b, c, d\}, \\
& \left(\begin{array}{llll}
0 & 0 & 1 & 1 \\
1 & 0 & 0 & 0 \\
0 & 1 & 1 & 0 \\
0 & 0 & 0 & 0
\end{array}\right) \delta_{6}=1, A / R^{\delta_{6}}=\{a, b, c, d\}
\end{aligned}
$$

In the equivalence classes of the attribute set: $A / R^{\delta_{1}}=\{a b c d\}, \quad A / R^{\delta_{5}}=\{a, b, c, d\}, A / R^{\delta_{6}}=\{a, b, c, d\}$ are either put into a single class or treated as a separate class therefore there is no practical meaning. So, for threshold values of $\delta_{2}=0.5, \delta_{3}=0.6, \delta_{4}=0.7$, the beset threshold $\delta_{3}=0.6$ can be identified using the $F$ statistics. With this threshold, the attribute set $A$ is classified $\{\{a\},\{b, c\},\{d\}\}$. Next, we will use congruence relation $T^{\delta_{3}}$ to find out the attribute power set $P(A)=\left\{\begin{array}{l}\{\phi\},\{a\},\{b\},\{c\},\{d\},\{a, b\},\{a, c\},\{a, d\},\{b, c\},\{b, d\},\{c, d\}, \\ \{a, b, c\},\{a c d\},\{b c d\},\{a b d\},\{a b c d\}\end{array}\right\}$

Its
$P(A) / T^{\delta_{3}}=\{\{\phi\},\{a\},\{b, c, b c\},\{d\},\{a b, a c, a b c\},\{a d\},\{b d, c d, b c d\},\{a c d$, $a b d, a b c d\}\}$. According to the reduction algorithm in attribute dependent space, the smallest set congruent to the attribute set $A$ is the attribute subsets $\{a c d\}$ and $\{a b d\}$, therefore both of them are the reduction of the attribute set. The absolute necessary attribute set i.e. core attribute set $\{a d\}$ can be found by calculating the intersection set.

In the end, we wish that this article can provide beneficial theoretical and applied contributions finding better, faster, more effective method of attribute reduction for the fuzzy information system .

\section{Acknowledgements}

This study was supported by the national natural science fund project(No.61203285),the education Department of Sichuan Province (No.14ZB0065), Key projects in Si
Chuan province department of education(No.12ZA138) and Science and Technology Project funds (No.K33).

\section{References}

[1] Liu Qing: Rough Inference 「M], Beijing Science Press, 2005(3): 20-67.

[2] Wang Reng, Miao Duoqian etc.: Summary of Theory of Rough Set and Its Application [J]. Pattern Recognition and Artificial Intelligence, 1996(6): 337-344.

[3] Zhang Wenxiu, Liang Yi, Wu Zhiwei: Information System and Knowledge Discovery [M]. Science Press, 2003.

[4] Novotny, M., Pawlak, Z. On a Problem Concerning Dependence Spaces [J]. Fundamental Informaticae, 1992 (16):275-287.

[5] Novotny M. Dependence Spaces of Information Systems [M]. In: Ortowska E.(ed) Incomplete Information Rough Set Analysis, Physica-Veolag, 1998:193-289.

[6] NovotnyM. On Dependence in Will's Contexts [J]. Fundamental information, 1993(19):343-353.

[7] E.Lois and M. Benedicty. Mappings Dependence Spaces [m]. Pittsburgh (Pennsylvania), March 1970.

[8] Cheng Tao, Huang Xiaokun: Attribute Reduction of Information System Based on Dependent Space [J]. Honghe University News, 2012(10): 26-28.

[9] Pawlak Z, Rough Sets [J]. International Journal of Computer and Information Science, 1982, 11(5): 341-356.

[10]Z.Pawlak: Rough Sets: Theoretical as Pects of Reasoning about Data [M]. Kluwer Academie Publishers, Boston, 1991.

[11] Andrew Kusiak*, Anoop Verma: Analyzing Bearing Faults in Wind Turbines: A Data-mining Approach [J]. Renewable Energy, 2012(48): 110-116.

[12]Alex Betphen J Smith: Data Warehousing, Data Mining, \&OLAP [M ] . New Yor k: McGraw - Hill Bo ok Co. , 1999. 